\title{
Celebration of Twenty Years from Dissolution Tester Equipment Manufacturers and Other Providers of Dissolution Related Services
}

\author{
Vivian A. Gray \\ Dissolution Technologies, Hockessin, DE
}

T his celebratory 20-year edition would not be complete without the comments from the manufacturers of dissolution equipment and other providers of dissolution related technical services or products. The comments below are listed in the order in which they were received. We appreciate this insightful feedback and, in some cases, historical perspective for 20 years of dissolution technology.

\section{Jensen Lee}

Logan Instruments Corp. www.loganinstruments.com

Logan Instruments Corp. was a late entrant into the dissolution tester manufacturing market. However, Logan had a large part in helping the industry grow. Automation, innovative concept design, and new equipment helped the industry flourish.

Logan Instruments Corp. was the first dissolution tester manufacturer to create the 8-position dissolution tester for automation in the U.S. market. The 8-position automated version consists of auto-lift, auto-sampling, and auto-dropping mechanisms via RS232 cable. Being user-friendly, the delivery head and sampling manifold can be moved up and down easily by pressing buttons or through a PC. For automated UV analysis, Logan's 8-position dissolution tester has six sample vessels, a blank, and a standard. The blank and standard vessels can also be used as preheated media replacement for automatic sampling systems. As the economic and production climate changed in the pharmaceutical industry, Logan created a 12-position dissolution tester. This unit helps customers in reducing their budget when a lab purchases one unit instead of two separate units, which also means less maintenance. Most of the generic drug companies need to mimic name-brand drugs by developing formulations. What's the best way to compare dissolution data side by side? The 12-position bath is perfect for this application. Logan is currently still the only U.S. manufacturer of this type of dissolution bath.

Continuing to be on the forefront of innovation in the dissolution industry, Logan developed the MDS-600 Media Delivery System and AVC-100 Automated Vessel Cleaner to reduce the downtime between dissolution tests. The MDS-600, which has been marketed since 1990, is still available with upgraded models that have current advancements. By its impeccable design, this unit can transfer six channels of precise, degassed, pre-heated media into six dissolution vessels simultaneously. With the success of the MDS, Logan introduced yet another first that makes the vessel cleaning process easy. The AVC-100 Automated Vessel Cleaner was introduced to wash the vessels while still in the dissolution tester; this helps limit the number of broken vessels. A vessel-cleaning method helps scientists to validate the cleanness of dissolution vessels. Both the MDS and AVC have increased the efficiency of the dissolution industry.

Looking forward, Logan pushed the limits by developing yet another new instrumentation, the Logan DISSO III-7 that combines both USP 3 and USP 7 testing methods, again for ease of operation and saving lab space. This instrument only needs to input the stroke length from USP 3 testing method to USP 7. The USP 3 method could be used for controlled-release drugs, which is to test drugs that dissolve within the Gl tract. The drug industry has improved through innovation and discoveries, so Logan has adapted to this process. Now drugs can be administered through the skin as well as implanted into the body. The USP 7 method can be used to test implants dissolving within the bloodstream and for quality control of transdermal patches.

With the knowledge of dissolution testing, Logan has crossed over into other fields in dissolution studies. Around 1995, Logan entered the transdermal field. Logan has taken the idea of drug testing through the skin with a Franz Cell to a new level. To purge the bubbles between membrane/skin and media has been a tedious job. With dynamic features, Logan's instrumentation provides a bubble-free system. In addition, Logan has automated this testing with several components that can run methods continuously for days as skin penetration can be administered over long periods of time.

Within recent years, Logan has engineered two product lines to satisfy these testing needs and has become the world leader in transdermal and stent/implant drug testing instrumentation.

\section{Werner G. Müller, CEO President of ERWEKA GmbH www.erweka.com \\ Congratulations to Vivian Gray for 20 years publishing Dissolution Technologies magazine.}


As the publisher of the standard magazine for dissolution testing technologies, Vivian Gray and her magazine strongly influenced and developed what nowadays is known as standardized modern dissolution testing regarding the scientific as well the technical approach.

The only magazine worldwide that specializes in dissolution testing, Dissolution Technologies is now known worldwide as THE source to turn to if you want to be up to date about latest developments and scientific approaches.

The idea of dissolution testing for research and for setting standards for the production of medications already started nearly 100 years ago! The first ideas on dissolution testing had been researched in the 1920s. For a long time, the idea of dissolution testing was done with disintegration testing equipment. In the 1950s the first idea to find a new way to get better results for dissolution of tablets was born. ERWEKA started developing the first dissolution apparatus according to the USP idea in the late 1960s.

My first personal contact with dissolution test apparatus was in 1977. At this time, a dissolution apparatus was still a simple stirrer unit for manual sampling. The standards among the several pharmacopeias were totally different. There were inch measurements, metering measurements, flat- and round-bottom vessels, and so forth. Every pharmacopeia worldwide had its own standards.

As the CEO of ERWEKA, I first met Ms. Vivian Gray in 1979 at the USP headquarters for some discussions about the different standards. Harmonization and standardization of the pharmacopeias was one of her greatest concerns and was very important to her. Over the years, we had many discussions at the USP to find solutions to get this harmonization, which nowadays is taken for granted, thanks to the never-ending efforts of the USP.

All these discussions and the requirements of the industry and the pharmacopeias, the demand for higher and higher standardization of the manufacturing process as well as quality control led the dissolution testing equipment from a simple stirrer unit to highly scientific equipment.

$U S P, B P$, and the $D A B$ harmonized to get the same standards. Metering measurements have been used. $B P, D A B$, and other European pharmacopeias have been harmonized in the EP. Other pharmacopeias worldwide followed. Today we have a worldwide pharmacopeial standard for dissolution testing equipment, which of course greatly helps us as a developer and manufacturer of advanced dissolution equipment to meet the worldwide requirements rather than produce several types of equipment for different specifications. This leads as well to comparable standards of the testing results of different equipment.

Manual sampling was followed by automatic sampling. Offline sample collection and later online UV-vis analysis of the dissolution tests followed! The next breakthrough for ERWEKA was initiated in 2006 by our most important customer, who wanted a fully automated system, which we developed in close cooperation. The result was the first RoboDis. In 2012 it was replaced by the second, even more advanced generation with full parallel approach to sampling and testing as well as being the first automated system $100 \%$ USP compliant apart from other enhancements.

Today, fully automated dissolution testing including automatic preparation of the test media, vessel filling, automated tablet drop, automatic parallel testing and sampling, online analysis by UV or HPLC, managed by advanced software with video recording and overlay of the dissolution curve, and even automated vessel cleaning can be done-all with one system and all fully automated for up to 40 batches. These heavy duty and high-volume dissolution systems managed by a robot hand now really work $24 / 7$ ! All conform $100 \%$ to USP/EP to ensure the same quality standards worldwide. And a scientific paper has shown that methods can be transferred from one RoboDis II system to another, even in different countries, showing the same results. This is dissolution testing on a whole new level.

For twenty years Dissolution Technologies has been the specialist scientific magazine to combine scientific and technical aspects! The target of testing tablets using the same standards should lead to better health and standardized medicine worldwide.

Thank you, Vivian Gray and the editorial board, for the great insight and valuable scientific research provided by your magazine to us on a regularly basis. For all her efforts and supportive work, we would like to present an ERWEKA award to Vivian Gray for 20 years of outstanding work in the field of dissolution technologies.

Vivian, I wish you all the best in continuing this great work for many more years to come!

\section{Scott Stephenson, Director of Operations Vortex Pharma Group, Inc. www.vortexsg.com}

\section{Dissolution-A Look Back at the Last 20 Years}

I would like to say thank you to Dissolution Technologies for the chance to give my perspective on the last 20 years of dissolution. For many like myself, the first time we saw a dissolution tester was at our first job. We were trained on how to use the system and then we ran test after test after test. Those of us in the dissolution equipment industry know that there is a lot that goes into design, development, and qualification to ensure the testers are rugged and reliable.

I have been in the dissolution qualification industry since 1998 when I went to work for VanKel Technology Group. At that time, many vendors had yet to establish validation groups to perform chemical testing or performance qualifications (PQs) on instrumentation. Most equipment companies provided only installation and operational qualifications, and even those few who would provide a PQ document rarely performed the chemical 
PQ test on site. Changes were already happening in the industry; the number of required calibration tests were reduced to four (Prednisone and Salicylic Acid, Apparatus 1 and 2) compared with the eight that had previously been required. Outsourcing dissolution calibrations to the instrument manufacturers became a very large business in the late 1990s and early 2000s. Today not only do instrument manufacturers calibrate their own equipment, but typically can calibrate any brand of dissolution tester. Also, the number of third-party vendors in the dissolution calibration field has increased dramatically since the early 2000s. These vendors provide a more neutral approach to dissolution calibration and have grown significantly as they offer a wider variety of multi-equipment calibrations.

When asked to write this article, I decided to share some of the hot topics from my early days that are still being talked about today in order to highlight where we were then and where we are today. Those new to dissolution may not (yet) have heard some of the stories below. And for others, you not only remember the stories, but probably still discuss them today.

The Detachable Paddle Blade Debate. Debate sparked quickly when one manufacturer decided to make a spinoff blade so customers could switch from paddle to basket without removing the shaft. A great idea; however, competitive companies wrote multiple articles and gave multiple talks about why this was not compliant. Today, almost every manufacturer makes detachable shafts.

Resident versus Non-Resident Probes. The concept was that a removable manifold that lowers and then raises (non-resident probe) has less impact on hydrodynamics than a resident probe. I am sure you can still "Google" the studies done at the time to test this, and I applaud all who know that they both could have an impact and need to be examined. This topic continues to be debated today.

Basket Clips. Some manufacturers use the three-clip ring to secure baskets as shown in the USP Apparatus 1 drawing. Others use an o-ring design. The topic of one being USP compliant and the other being non-compliant existed in the calibration world for years. While there were several studies that showed a small difference in Prednisone averages with three-ring versus o-ring, once again almost every manufacturer now offers an o-ring style basket assembly. Some even offer the three-ring clip, not based on its functional purpose, but just as an "add on" to make it exactly like the USP drawing. This discussion still goes on today.

In conclusion, I guess the best way to sum up the last 20 years for dissolution is that we all continue to improve. Equipment manufacturers continue to develop and improve equipment that helps eliminate or reduce variability in dissolution tests. Prednisone calibration tablets have become much more reproducible and discriminatory. ASTM mechanical calibration has become a sound alternative for companies who want to move away from the Prednisone calibration test. Dissolution continues to be dynamic in nature, and industry professionals, equipment manufacturers, and service providers all continue to discuss, debate, and evolve. The controversies and differences that we have shared over the last 20 years have provided many changes that I feel have made the field of dissolution better. I hope to be around twenty years from now to talk about all the interesting, new dissolution equipment and related services. And I bet that some of the topics debated 20 years ago and today will still be up for discussion then.

On a personal note, I wanted to say thank you to a person who was instrumental to my career in dissolution and to the industry itself. Jim Swon, previously the owner of VanKel Technology Group, was an amazing man who taught me many things about dissolution, business, and life. Jim, who passed away in September 2013, will be missed by all who knew him, and I would not be writing this article today had it not been for the enthusiasm Jim cultivated in me about the dissolution industry.

\section{Royal Hanson \\ Hanson Research Corporation www.hansonresearch.com}

I can remember over twenty years ago when Cynthia Brown and James Oldham contacted me with a dream of starting a journal dedicated to the science of dissolution testing. Hanson supported their efforts from the beginning, and my dad, Bill Hanson, wrote an article on dissolution for the inaugural issue of Dissolution Technologies in 1994. In fact, Hanson was the only manufacturer willing to support their efforts in getting this project off the ground.

Vivian Gray has since taken over as Managing Director. Now celebrating its twentieth anniversary, Dissolution Technologies has grown into a world-class industry journal disseminating relevant and timely information on all aspects of dissolution testing. Its pages have reflected the evolution of this scientific discipline that continues to promote quality assurance in the pharmaceutical industry.

As the literature has grown, so have the technology and its application. Dissolution equipment has evolved these last twenty years into the precision test instruments that we know today. Capabilities have expanded to include a full array of automation, including media prep, sampling, filtration, collection, and online analysis. Computer and information technologies have benefited the analyst with both enhanced instrument control and data management.

Global industry trends, regulatory concerns, and instrument qualification requirements have impacted the technology. A host of industry guidances, such as Biopharmaceutics Classification System (BCS), Process Analytical Technology (PAT), and Quality by Design (QbD), has elevated the science of dissolution during this time. The ASTM standard E2503-07/13 has emphasized a rigorous mechanical calibration of testers. The Reduction of 
Hazardous Substances (RoHS) directive has also resulted in more Earth-friendly technology.

The USP has played an ongoing role in promoting international harmonization in technology and apparatus. USP has updated and refined its general chapters, addressing the requirements of dissolution testing, and has introduced new chapters on relevant topics such as product quality tests, analytical instrument qualification, method development, and validation. A host of international symposia now focuses on the science of dissolution testing and its global ramifications, including AAPS, FIP, Analytica, CRS, and others.

As we look to the future, we may anticipate continuing innovation and discovery in medicine and global health care. I believe that our industry will continue to provide rugged and robust quality assurance tools and practice to support this effort. I find this an exciting time in dissolution testing, and I look forward to the future. Congratulations to Dissolution Technologies on twenty years of excellence!

\section{Robert Spock, Bruce Renslow, and Raymond Majeski Quality Lab Accessories, LLC www.qla-Ilc.com}

QLA has been a leading supplier of dissolution accessories and consumables for over 17 years. USP $<711>$ sets forth requirements that were established decades ago. Since that time, dissolution accessories and instruments have become available that far exceed the requirements of USP $<711>$. However, it has not been updated to reflect the current capabilities of the industry.

Let's consider a few of USP $<711>$ requirements:

- Dissolution Vessels: The vessel "inside diameter is 98 to $106 \mathrm{~mm}$."This allows vessel diameters to vary by $8 \mathrm{~mm}$. How much coefficient of variation to dissolution rates might this cause? Articles have been written to illustrate the answer. Currently, there are a few vessel suppliers that offer precision molded vessels with inside diameters held to a tolerance of $\pm 0.20 \mathrm{~mm}$.

- Paddle and Basket distance from the inside bottom of vessel "is maintained at $25 \pm 2 \mathrm{~mm}$ during the test." This requirement is easily set within $\pm 0.5 \mathrm{~mm}$.

- Wobble:"The shaft... rotates smoothly without significant wobble...." How much wobble is significant?

- Vibration specifications and tolerances need to be well defined.

These are just a few examples, but unfortunately there are many more. Until USP $<711>$ is updated to better define these specifications and tolerances, we need not be surprised that ASTM E2503-07 Enhanced Mechanical Calibration has offered a logical alternative.

At QLA, we believe that the most critical change to the U.S. dissolution industry has been happening over the past five years:
Enhanced Mechanical Calibration versus Performance Verification Testing (ASTM E2503-07) versus USP $<711>$

New dissolution instruments have been designed to achieve the Enhanced Mechanical Calibration specifications required by ASTM E2503-07. At the same time, greatly improved and innovative dissolution accessories that permit older dissolution instruments to meet and exceed these stricter mechanical requirements are now available. This is possible due to the emergence of more advanced and technically precise manufacturing equipment, methods, and tools for validation of those methods. As a manufacturer of dissolution accessories, we have experienced many benefits in both efficiency and product quality from technological improvements to machinery, materials, and processes over our company's history.

PVT and the resulting acceptance criteria set forth rely on reference standard tablets that have collectively been validated by a method that uses numerous laboratories and standard dissolution instruments. This practice seems to go against guidelines set forth by the National Institute of Standards and Technology (NIST). In simple terms, equipment used to calibrate the accuracy of any measuring device must be far more accurate than the device being calibrated. NIST provides specific formulas for meeting this requirement. Reference standard tablets have not been validated in such a manner and therefore do not meet this NIST requirement. Proper validation of reference standard tablets should be accomplished on dissolution instruments that meet extremely tight mechanical specifications. Although there has never been any attempt to do so, it could be done. Until true dissolution release rates are determined on a "perfect" instrument, it seems as though reference standard tablets will not produce meaningful results. In fact, questionable reference standard tablets may result in calibration failure of excellent dissolution instruments. Troubleshooting calibration failure can be time consuming and costly. Mechanical specifications can be easily rechecked and confirmed for compliance; however, the most critical part of the test, the reference standard tablet, cannot. Thus, Enhanced Mechanical Calibration continues to gain popularity in pharmaceutical laboratories.

\section{The Future of Dissolution}

Additional enhancements to Enhanced Mechanical Calibration are possible and likely to become reality. More laboratories will add them to their current calibration routines and ultimately abandon PVT. Accurate dissolution instruments and accessories will provide more accurate and precise dissolution results. Our observations have shown that this trend will continue to increase in the future. Reference standard tablets could be useful, but until they conform to NIST standards, they may contribute more to the problem than the solution. QLA will continue to design and bring the highest quality innovative dissolution products to our customers at cost-effective prices. 


\section{Kevin Whitkanack Sotax Corporation www.sotax.com}

\section{Too-Many-Samples Syndrome}

Included in the 1994 issue of Dissolution Technologies was an article we had written about a syndrome dubbed "Too Many Samples." In all that has changed in the industry since then, we still haven't found a cure for the insatiable sample load required during formulation and release testing of pharmaceutical dosage forms. In fact, with initiatives like QbD and Continuous Processing, the sample load has only increased, especially for the groups responsible for analytical formulation support.

Cutting-edge formulations, while advantageous for the consumer, introduce new challenges for dissolution method developers. We have therefore continued to expand our platforms to allow for the flexibility needed by these teams. While robustness of instrumentation is as important as ever, the need to alter the tool to the task has become a leading requirement for many. Our fully automated systems have added several features for this purpose such as low volume dissolution, use of multiple media types for media screening, and the ability to use fewer than six vessels at a time for those precious early phase samples. The support of automation during Design of Experiment (DoE) work is very beneficial when the instrumentation can elegantly alter various test parameters. With engineering and application science centers in Massachusetts, Switzerland, and Mumbai, SOTAX has grown into an organization that is uniquely positioned to develop and support solutions for pharmaceutical testing for customers around the world.

For much of the last twenty years, SOTAX and Zymark had worked independently to offer full automation for dissolution and automated sample preparation. Several iterations of the Zymark MultiDose and SOTAX AT70 afforded the instruments enhanced robustness as the feature set quickly grew to support the change in test methods. Our lab automation in the early 90 s was converting from $\mathrm{XYZ}$ task-performing robots to specialized systems for high-throughput QA/QC labs. SOTAX and Zymark joined forces in 2008, and the engineering teams have been able to work together to launch the now 4th generation MultiDose as well as an entire new Xtend ${ }^{\mathrm{TM}}$ dissolution line. These new platforms were built on the premise that many dissolution groups today are in need of a modular, scalable system.

Several new product types have required creative techniques for monitoring drug release characteristics. It's always impressive to visit customers or attend conferences and see the powerful new ways to study novel dosage forms. Apparatus 4 plays an active role in IVIVRT for products like semi-solids, microspheres, and nanoparticles. New flow-through cell inserts such as the A4D developed by the University of Connecticut have helped define the test for liposome-based formulations. Flow-through dissolution continues to find more applications in areas as diverse as small-volume dissolution and long-term elution studies such as those required for implants. The technique has also proved to be very useful at detecting formulation subtleties that are below the sensitivity threshold of Apparatus 1 and 2. Because of the need for advanced dissolution method development, SOTAX created a separate CRO organization, SPS Pharma Services, in 2005. SPS is a unique $\mathrm{CRO}$ in that they are the only outsourcing company dedicated to dissolution testing. The lab has recently moved into a larger facility in Orleans, France and is now a fully accredited GMP CRO.

In 2007, the FDA published "Standard Practice for Qualification of Basket and Paddle Dissolution Apparatus" (ASTM E2503-07) and later issued a corresponding Industry Guidance on "The Use of Mechanical Calibration of Dissolution Apparatus 1 and 2-Current Good Manufacturing Practice (CGMP)." With these publications, the agency took the position that it is acceptable to qualify a dissolution apparatus based solely on a verification of certain mechanical parameters. Mechanical calibration has significantly increased the demand for precision manufactured, "auto-compliant" dissolution testing equipment that passes stringent mechanical procedures without the constant need for adjustment or calibration. To facilitate the mechanical qualification process, SOTAX has developed a Mechanical Qualification Device (MQD) that can be used to qualify all major brands of dissolution testers. The MQD greatly improves the quality of the mechanical measurements and streamlines the reporting of the results into a concise calibration report.

The cell phone analogy is an often used to describe technology changes since the mid 90s. Life without the cellular tether is our "uphill both ways" story to today's youth. The equipment used for dissolution obviously didn't change quite as drastically, but there are some major advantages to being able to build with today's toys. The electronics and software used to control automation has improved tremendously. Robotic positioning now easily compensates for drift, and software continues to handshake with other equipment and LIMS systems to ease the burden and compliance risk associated with data tracking. The lines between firmware and software are blurring with the ability to control formerly complex systems with elegant capacitive glass touchscreen displays. Many have added video monitoring options including our CenterView $^{\mathrm{TM}}$ video monitoring that assists in both formulation development and investigation support.

Real-time release has, in a number of filings, managed to greatly reduce the need for traditional dissolution/assay testing for product quality assessment. It has not however solved the "Too Many Samples" problem. Many samples are still tested upstream to build and validate spectroscopic models, and many samples are still run to support the ever more complicated task of formulation screening. 
With the acquisition of Pharmatron, we've taken an active role in the RTR production environment with the Tandem III NIR module for connect-to-press assay/CU. We'll continue to support the changing goals of groups in this arena by handshaking our efficient physical testing solutions in production with dissolution and assay testing equipment for enhanced process validation activities. Our goal as a manufacturer is to be able to provide flexible solutions and reliable service to our customers. We'll be active in supporting industry initiatives for workflow integration and look forward to continuing our partnerships for the next 20 years and beyond.

\section{G. Bryan Crist \\ Scientific Affairs Manager, Dissolution Systems Agilent Technologies, Inc. www.agilent.com}

When we look at the dissolution environment for dosage performance testing, we see that it's quite simple and consists of three main components: a vessel, medium, and an agitation source. For such a simple concept, you have to wonder how individuals can write countless articles, produce numerous workshops, deliver years of presentation material, and have a magazine dedicated to dissolution topics du jour for twenty years. So there is obviously something quite complex about dissolution and drug release testing in a pharmaceutical production environment where the only thing that is consistent is change. We do, in fact, need an avenue to connect current technology to the innovative dosage forms of the age. Congratulations Dissolution Technologies; you have kept us well educated and delivered a forum for new technology, techniques, regulatory guidance, and compendial resources, and I can only hope it will continue well into the future.

In 1994 VanKel instruments were shown in the early issues of Dissolution Technologies along with several other dissolution manufacturers. As times have changed for the pharmaceutical industry, VanKel has also evolved through integration into Varian in 2001 and then again into Agilent in 2010. Agilent's level of engineering, manufacturing, and service is unsurpassed in the life science analytical instrument business, and the dissolution systems business has benefited greatly from this integration. As a compliment to Dissolution Technologies, readers can not only keep up to date with changes in dosage forms and analytical method development, but they can also keep abreast of the product offerings that instrument manufacturers supply.

While I have enjoyed providing articles for Dissolution Technologies from time to time, I have gained an immeasurable respect for the authors of so many diverse topics represented in this periodical. The managing editors through the years, Cynthia Brown and Vivian Gray, have routinely sought authorship on articles covering all aspects of dissolution and drug release testing, from benchlevel testing tips to ongoing dynamic research, innovative technologies to methods for novel dosage forms. All the articles are peer reviewed and provide consistent quality in their content.

As a manufacturer in a very niche industry, Agilent is fortunate to have a targeted periodical where we can keep dissolution scientists up to date with our most current apparatus, automatic sample collection equipment, online systems analytical systems, and tools for qualification and calibration. In addition, Dissolution Technologies provides a platform for Agilent to promote training programs and seminars, webbased resources, our extensive Dissolution 1-on-1 Training Course, the Agilent-sponsored Dissolution Discussion Group (DDG), and other global tools to ensure dissolution scientists are well equipped for their laboratory work.

The Agilent dissolution business team appreciates our partnership with Dissolution Technologies and looks forward to future collaboration. Congrats, DT!

\section{Gerry Brinker \\ Chairman, Distek, Inc. www.distekinc.com}

\section{Dissolution Testing-A Twenty Year Retrospective}

During the past twenty years the "Dissolution Test," as described in USP $<711>$, has progressed with some modest modifications and improvements. In the same period, the instruments used to perform these tests have gone through major changes and enhancements.

In the early 1990s Distek developed the 2100 Series dissolution bath, which incorporated some novel features. Permanent shaft centering, vacuum-formed one-piece water baths, extremely accurate temperature and rpm control, vessel covers that significantly minimized media evaporation, and ease of operation were all considered cutting-edge technology at the time. Right from the start, the major focus of our engineering team was to design instruments with unsurpassed control of the test variables and uncompromising ease of setup and use. A testament to Distek's tenacity can be found in many of today's laboratories that still use our 2100 Series test instruments.

Trying to innovate in the dissolution marketplace has never been easy. How does one take a rather simple electromechanical instrument (i.e., motor, rpm controller, water bath, and bath temperature controller) and have it stand out from competitive products? The weakest link was always the water bath. Algae formation, possible vibration from thermo circulators, and potentially inconsistent temperatures in different parts of the bath were all issues waiting to be resolved.

Prior to 1996, dissolution testers were all water bathbased devices. Temperature control of the vessels was maintained by a water bath and thermocirculator. We decided it was time to develop a more technically advanced system that could heat the media using heater jackets and maintain the temperature using sensors embedded in the rotating shafts. This gave rise to the first "bathless" dissolution tester, the Premiere 5100. 
Instead of controlling temperature from a single location in the bath, we were now able to focus on the actual media in the vessels, since each position could now be independently controlled. Instead of monitoring media temperature periodically by lowering an external temperature sensor with potential hydrodynamic disturbances, we were now able to provide a more elegant solution. The embedded sensors reported and controlled the temperature continuously, thus acting as six solid-state mini baths as opposed to one large water bath. Additionally, we could now provide a complete history of vessel temperature as well as rpm. Especially for long term, sustainedrelease testing, this turned out to be quite significant.

As in any new innovative product design, our initial efforts were not perfect, but with tenacity and perseverance, we succeeded and introduced the Premiere 5100 to the marketplace in 1996. Almost immediately, the new technology generated a lot of curiosity. However, disruptive technologies are rarely accepted universally. Distek was fortunate to be involved in the pharmaceutical industry, whose scientists were always curious and accepting of novel approaches to old problems. As such, the 5100 became successful almost from the very start.

A few years later, improvements were made to the hardware and firmware, which resulted in the release of the second generation "bathless," the Model 6100. During this period, we came to realize that our new concept would not be accepted universally and that we shouldn't put all our eggs into one basket. We decided to extend the capability of the 6100 by adding a water bath but maintained the individual vessel temperature advantages associated with the embedded shaft sensors. The water bath could still be controlled based on the temperature in the vessels rather than that in the water bath.

During this same period, significant developments took place in the area of automation. Fully automated systems, both robotic and fixed, became available and permitted multiple dissolution tests to be performed unattended. They were generally expensive and required time-consuming validation protocols but were most useful for high-volume testing of rapid-release dosage forms. Distek opted to take a different approach.

We focused on developing instruments that were convenient and time saving at the beginning and end of the test. A media preparation system was introduced which dispensed preheated, deaerated and accurately measured volume directly into each vessel. A vessel-washing instrument was also developed which precluded the need to remove vessels at the end of the test for emptying and cleaning. Since the vast majority of dissolution tests are performed using individual test units, the ability to automate media preparation and vessel cleaning turned out to be quite desirable.

At the same time, advanced sample collection systems were developed to eliminate the need to collect aliquots manually for downstream analysis by UV or HPLC. Our Model 2230 was a peristaltic pump based collector that was able to sample from six or twelve vessels simultaneously. Later, the Evolution 4300 was developed to address customer requirements for collecting volumes more accurately. A syringe pump that was capable of sampling through finer syringe filters was used for this purpose. Running two different methods simultaneously was a unique feature offered by the 4300 that went well beyond customer requirements and expectations.

Online UV systems were also becoming popular, especially for extended-release dosage forms. The ability to perform a test and have the dissolution profile generated at the end proved highly desirable. Later, online HPLC systems arrived on the scene as well. The industry developed to the point where there was no longer a need to sample manually, unless cost was an issue or the test only required one or two time points.

The new millennium saw heavy competition between conventional photodiode array automated UV systems and a more advanced technology that used optical fibers and CCD cameras. Always rising to the needs of the market's competitive climate, Distek popularized the Opt-Diss 405 , which was capable of monitoring six or twelve vessels simultaneously. Using custom software to conveniently track dissolution profiles in real time, stationary small-diameter fiber-optic probes residing in each vessel reduced hydrodynamic interferences and eliminated the need for media replacement.

A few years ago, we decided to focus on the next generation of dissolution testers. As in previous design cycles, it was difficult to envision not only what would be novel, but also how it would advance the dissolution experience. Most conventional devices used a single-drive motor and one or more belts to drive the spindles and shafts; however, motor technology had advanced significantly, and we were able to design a totally unique concept. The new system used a small, single-drive motor for each vessel and spindle position. In addition to driving the paddles or baskets during the test, it was also designed to automatically raise and lower each shaft at the appropriate time. Using this modular concept, our new Symphony 7100 gave users the ability to test up to three different products at one time. We also spent considerable development time on the user experience by introducing a color touch screen capable of a simple yet advanced graphical user interface.

So, what can we expect over the next twenty years? Predictions are always difficult, but one thing is certain: dissolution instruments will look and feel vastly different from what they are today.

\section{Johannes Krämer \\ PHAST GmbH \\ WWW.PHAST.com}

\section{Best wishes from PHAST in Germany}

PHAST, as one of the leading partners in dissolution testing, would like to congratulate Dissolution Technolo- 
gies for successfully bringing together the dissolution community over the past 20 years!

The conception of PHAST is a continuation of dissolution activities that were started by Helga Möller in the Central Laboratory of German Pharmacists, ZL. Helga Möller was the head of ZL's biopharmacy group in Eschborn. This is where the Langenbucher flow-through cell apparatus was successfully promoted into the European Pharmacopoeia. It wasn't harmonized at that time but was still under ZL's responsibility. Johannes Krämer continued the effort that was more than 20 years embedded in the activities of FIP's (Fédération Internationale Pharmaceutique) Dissolution Working Group. His dissolution lab was part of the management buyout he had initiated. Dissolution and drug release activities are an integral part of the services provided initially by his company called LQS. Then moved to Homburg, the company was renamed PHAST (Pharmaceutical Quality Standards). With this genetic code, the path toward "In Vitro Dissolution" and "Drug Release Testing" was preprogrammed.
In addition to USP 3 and USP 4 apparatus, there are over 70 USP 1 and USP 2 apparatus in service at PHAST at present. These are mainly used for batch release testing and the development of dosage forms. In terms of volume, oral dosage forms are in the foreground, along with the increase in testing for those products, which is applied by alternative routes of administration.

PHAST is proud to have installed all dissolution apparatus that has been described in the international harmonized pharmacopoeia (USP, Ph. Eur., JP) in the qualified GMP environment.

With the increasing trend to implement biologics in therapy, PHAST develops innovative drug release testing methods for this new class of drugs and the associated dosage forms, such as nanoparticles, in its Dissolution Center of Excellence. In this scientific field, PHAST is continuously cooperating with universities and other research organizations, resulting in a considerable number of scientific publications.

PHAST feels strongly committed to the USP and is pleased to organize regular dissolution training courses of the USP in Germany. 